\title{
A Study of Two-dimensional Barcode Prescription System for Pharmacists' Activities of NHI Contracted Pharmacy
}

\author{
Wei-Ling WANG ${ }^{*, a}$ and Chyi-Hong $\operatorname{LIN}^{b}$ \\ aDepartment of Industrial Engineering and Management, National Chin-Yi University of Technology, 35, \\ Lane 215, Section 1, Chung-Shan Road, Taiping City, Taichung County 411, Taiwan and \\ ${ }^{b}$ Information Process Committee of Taichung City Pharmacist Association (TCCPA), \\ 3 of 5 Floor, Section 1, Taichung-Kang Road, Taichung 403, Taiwan
}

(Received July 7, 2007; Accepted September 26, 2007)

\begin{abstract}
To lower the cost and enhance the quality of healthcare service, the Taiwan government launched National Health Insurance (NHI) in 1995 and the System of Separation of Medicine and Pharmacy in 1997. After the separation of dispensing and prescribing (SDP), pharmacists in NHI-contracted pharmacies have the right to fill prescriptions from a clinic. This means that pharmacists in NHI-contracted pharmacies have four more activities than previously. How to improve the additional activities remains an issue to be solved. Today, in most countries, patients deliver a written prescription to the pharmacy of their choice. The pharmacist validates the prescription and dispenses the drug. In this paper, we describe an automatic data collection system, the two-dimensional barcode prescription system (2DBPS) for pharmacists. The system allows patients to deliver a paper prescription with a 2D barcode issued by a clinic to the pharmacy of their choice. The pharmacist scans in the 2D barcode, validates the prescription, and dispenses the drug. Evaluation of the 2DBPS showed that most pharmacists use it to execute additional activities and it was more efficient than before. Moreover, the easy-to-use 2DBPS is accepted and appreciated by pharmacists. We can thus conclude that the aim of the 2DBPS is to reduce the workload of pharmacist in data processing. It will help pharmacists to expand their role beyond simple dispensing and data processing to providing pharmaceutical care services.
\end{abstract}

Key words—— two-dimensional barcode; prescription; pharmacists' activity

\section{INTRODUCTION}

A National Health Insurance Program (NHIP) has been operating in Taiwan since March 1995 when the government changed its policy to integrate individually managed insurance programs targeted at labor, public employees, and the agricultural sector. About $57 \%$ of the group insurance population was in the three sectors at that time. By the end of 2004, NHIP included nearly $99 \%$ of the insured in Taiwan. ${ }^{1)}$ Two years after the beginning of the NHIP, the government began to push for the separation of dispensing and prescribing (SDP) of pharmaceuticals in March 1997. By the end of 2006, community pharmacies totaled 7397, and the NHI had contracted 4036 as special agents in carrying out its plans. Community pharmacies comprise about $55 \%$ of all pharmacies. ${ }^{2)}$

Before the execution of SDP pharmacists' activities in community pharmacies mainly included retail sales of over-the-counter (OTC) preparations, management of OTC preparations, verification of medicine,

\footnotetext{
*e-mail: wlwang@ncut.edu.tw
}

and guidance of patients on drug compliance. After SDP, pharmacists in NHI-contracted pharmacies have the right to fill prescriptions from clinics. Pharmacists' activities in NHI-contracted pharmacies are more numerous than those in community pharmacies. The additional activities are: dispensing prescriptions, checking prescriptions, management of prescriptions and prescription drugs, and prescription filing. More data must therefore be processed than before in NHIcontracted pharmacies. How to increase the performance of pharmacists in NHI-contracted pharmacies is a new issue in pharmaceutical science in Taiwan.

In the "post-info age," researchers in the field of pharmainformatics must develop software for pharmaceutical sciences as well as applications to pharmaceutical data to assimilate the greatest quantity of hardware. Otherwise, pharmaceutical scientists will not be able to enjoy fully the benefits of information technology. ${ }^{3)}$ Automatic data collection (ADC) systems are defined as "the electronic means of collection raw data input to be processed and analyzed in order to assist management decision making." Benefits of ADC systems include improving the ac- 
curacy of the data, making data more rapidly available to those who need them, and improving job performance. ${ }^{4)}$ Two-dimensional (2D) barcodes are part of ADC technology. They store more information than one-dimensional (1D) barcode. 2D barcodes function as a portable data file and entire data records. The required information is contained in the symbol itself. This can be text, photographs or signatures, or a combination of the three. 1D barcodes are only a primary key code to access the database for detailed information. Without an external computer system, the 1D barcode has no meaning as there is nothing for the keys in the barcode to unlock. ${ }^{5)}$ Medical applications for 2D barcodes include blood bag identification $^{6)}$ and biometric identification. An example of biometric identification is the Biometric ID Project of the state of Connecticut in the USA begun in January 1996. The state legislature passed a law requiring that Aid to Families with Dependent Children (AFDC) and General Assistance recipients be biometrically imaged for identification purposes. A tamper-proof, secure photo identification card is containing the applicant's photo, welfare identification number, 2D barcode containing fingerprint data for identification verification, and medical eligibility data for medical service providers is issued. ${ }^{7)}$

In this paper, we describe a $2 \mathrm{D}$ barcode prescription system (2DBPS) for use by NHI-contracted pharmacies to improve the performance of additional pharmacists' activities after SDP. The system allows patients to deliver a paper prescription with a $2 \mathrm{D}$ barcode issued by a clinic to the pharmacy of their choice. The pharmacist scans in the 2D barcode, validates and dispenses the drug, and then the prescription data is stored in the 2DBPS database for other additional activities. For the NHIP, the pharmacist needs to upload the following refill prescription data to the NHI: 1) healthcare unit ID;2) healthcare unit name; 3) fee-related diagnostic category; 4) patient name; 5) patient social security number; 6) patient date of birth; 7) consulting department; 8) appointment date; 9) NHIP ID card control number; 10) days of medicine prescribed; 11) diagnosis; 12) physician ID; 13) medicine name; 14) medicine dose/use; and 15) total quantity. Until the prescription is filed by the NHI-contacted pharmacy, healthcare insurance reimbursement is not be paid by the Bureau of National Health Insurance (BNHI). The 2DBPS can assist the pharmacist to execute additional activities.
A study to compare the additional pharmacists' activities before and after introducing 2DBPS showed that the introduction of this system improves the additional activities of pharmacists. Moreover, the results of evaluation on ease of use were favorable. The present study aims to reduce the workload of pharmacists' activities in data processing. It will help pharmacists to change their role beyond simple dispensing and data processing to that of pharmacists who can provide pharmaceutical services.

\section{METHODS}

One of the aims of the 2DBPS is to improve pharmacists' additional activities after SDP. To evaluate whether it achieves this, we conducted a questionnaire survey of pharmacists in NHI-contracted pharmacies in central Taiwan from October to December 2006. The survey questionnaire consisted of two parts: pharmacist consent to participate in the survey including use of the 2DBPS, questions prior to using the 2DBPS (pre test), and questions after using the 2DBPS (post test). In addition to pharmacists' activities, the post test questions included three questions to evaluate the ease of use of the 2DBPS. To investigate improvement of pharmacists' additional activities after SDP, there were four identical pre and post test questions: 1) dispensing of prescriptions is efficient; 2) checking of prescriptions is efficient; 3) management of prescriptions and prescription drugs is efficient; and 4) prescription filing is efficient. The reasons for efficiency are data accuracy, timeliness, and labor reduction. Pharmacists in NHI-contracted pharmacies were randomly selected from a list for their additional activities. During the survey, trained assistants were available onsite to help selected pharmacists operate the system. Pharmacists who participated in the study were asked to answer the pre test questions before using the 2DBPS. The assistant then arranged for them to use the system, after which they completed the post test questions. Because the survey questionnaire was performed onsite, 90 pharmacists participated in the study and 90 survey questionnaires were returned, for a recovery rate of $100 \%$.

The response to each question was on a five-point Likert scale: completely agree (5); agree (4); OK (3); disagree (2); and strongly disagree (1). The meaning of "OK" is neither agree nor disagree. Through the scale, we identified which additional activities we are improved before and after. For data 
reliability, we also examined the internal consistency of each question factor by calculating Cronbach's coefficient $\alpha$, which determines the extent of relatedness between question items and expresses the internal consistency of the scale as a whole. The statistical analysis package SPSS10.0 for Windows was used for all statistical processing. ${ }^{8)}$

\section{RESULTS}

Reliability Analysis Table 1 shows the extent of relatedness between question items and the internal consistency of the scale in the survey. Nunnally and Bernstein suggested that " $\alpha$ " should be at least equal or greater than 0.7 to have acceptable reliability. ${ }^{9)}$ In Table 1 , the $\alpha$ values are all greater than 0.7 , and hence the survey results had high reliability.

Evaluation of Pharmacists' Additional Activities The results of pharmacists' additional activities status before and after the use of the 2DBPS are shown in Table 2. After using the 2DBPS, most pharmacists (about 84\%) agreed or strongly agreed that they were able to check prescriptions efficiently. Similarly, most pharmacists (about $88 \%$ ) agreed or strongly agreed that after using the 2DBPS they were able to manage prescriptions, prescription drugs, and prescription fil-

Table 1. Reliability Analysis

\begin{tabular}{cccc}
\hline \hline Question & Sample size & No. of questions & $\begin{array}{c}\text { Cronbach's } \\
\text { coefficient } \alpha\end{array}$ \\
\hline Pre test & 90 & 4 & 0.7436 \\
\hline Post test & 90 & 4 & 0.7215 \\
\hline
\end{tabular}

ing efficiently. Fewer pharmacists (about 21\%) agreed or strongly agreed that they were able to dispense prescriptions efficiently. However, most pharmacists (about $71 \%$ ) said OK, agreed, or strongly agreed that after using the 2DBPS they were able to dispense prescriptions efficiently. This supports the view that the 2DBPS allows pharmacists to perform additional data processing activities. Data processing is any computer process that converts data into information or knowledge. Before data processing, hand written or printed records had to be typed into a computer in a process called data entry. In NHI-contracted pharmacies data entry and data processing are required by pharmacists.

To investigate the dependency of results before and after the use of the 2DBPS, we adopted Wilcoxon signed-rank test with a $99 \%$ (i.e., $\alpha=0.01$ ) confidence interval to calculate the statistical significant difference between the two results. Table 2 shows that the survey sample data before and after the use of the 2DBPS are independent.

Ease of Use of the 2DBPS There are at least three factors to consider when evaluating the ease of use of the 2DBPS: document navigation; system manipulation; and usefulness. The results are shown in Table 3. Most pharmacists (78.89\%) agreed that they were able to process the prescription data they wanted easily. Most considered the system easy to use $(82.22 \%)$ and found the 2DBPS useful (84.44\%). Very few patients (less than 1.22\%) had negative views of the 2DBPS. Based on the survey results, we believe that most pharmacists will accept the 2DBPS.

Comparison of Additional Activities before and af-

Table 2. Results before and after the Use of the 2DBPS $(n=90)$

\begin{tabular}{|c|c|c|c|c|c|c|c|}
\hline Question & $\begin{array}{l}\text { Pre/post } \\
\text {-test }\end{array}$ & $\begin{array}{l}\text { Strongly } \\
\text { agree } \\
(\%)\end{array}$ & $\begin{array}{c}\text { Agree } \\
(\%)\end{array}$ & $\begin{array}{l}\text { OK } \\
(\%)\end{array}$ & $\begin{array}{c}\text { Slightly } \\
\text { disagree } \\
(\%)\end{array}$ & $\begin{array}{c}\text { Disagree } \\
(\%)\end{array}$ & $\begin{array}{l}\text { Wilcoxon } \\
\text { signed- } \\
\text { rank test } \\
(\mathrm{Z})\end{array}$ \\
\hline \multirow{2}{*}{ Dispensing of prescriptions is efficient } & Pre & 3.33 & 11.11 & 47.78 & 31.11 & 6.67 & \multirow{2}{*}{$-3.358^{*}$} \\
\hline & Post & 6.67 & 14.44 & 50.00 & 26.67 & 2.22 & \\
\hline \multirow{2}{*}{ Checking of prescriptions is efficient } & Pre & 1.11 & 5.56 & 7.78 & 42.22 & 43.33 & \multirow{2}{*}{$-7.947^{*}$} \\
\hline & Post & 53.33 & 31.11 & 11.11 & 3.33 & 1.11 & \\
\hline \multirow{2}{*}{$\begin{array}{l}\text { Management of prescriptions and } \\
\text { prescription drugs is efficient }\end{array}$} & Pre & 1.11 & 1.11 & 12.22 & 35.56 & 50.00 & \multirow{2}{*}{$-8.151^{*}$} \\
\hline & Post & 48.89 & 38.89 & 10.00 & 1.11 & 1.11 & \\
\hline \multirow{2}{*}{ Prescription filing is efficient } & Pre & 3.33 & 4.44 & 8.89 & 37.78 & 45.56 & \multirow{2}{*}{$-7.904^{*}$} \\
\hline & Post & 50.00 & 37.78 & 7.78 & 3.33 & 1.11 & \\
\hline
\end{tabular}

\footnotetext{
${ }^{*} p<0.01$
} 
ter Use of the 2DBPS Figure 1 shows the comparison of additional activities before and after the use of the 2DBPS. After using the 2DBPS dispensing of prescriptions was only slightly more efficient $(6.67$ $\%)$. However, the 2DBPS made checking prescriptions much more efficient (77.77\%). Similarly, management of prescriptions and prescription drugs was $85.56 \%$ more efficient. Prescription filing was also easier $(80.01 \%)$. Dispensing of prescriptions with the 2DBPS focuses on manual operations for dispensing but required less data processing. The pharmacists almost have automatic machine for drug dispatching and package. This will reduce the difference between before and after use of the 2DBPS. From Fig. 1 we can conclude that the 2DBPS can really help pharmacists to improve the additional activities.

\section{DISCUSSION}

To transfer prescriptions directly to the pharmacy using either fax or EDI limits the patients' freedom of choice in selection of a pharmacy. ${ }^{10)}$ Under the SDP program, after a patient leaves a clinic, he or she can approach any NHI-contracted pharmacy to fill a prescription. The 2DBPS allows patients to deliver a

Table 3. Results of Evaluation of Ease of Use of the 2DBPS $(n=90)$

\begin{tabular}{lccc}
\hline \hline \multicolumn{1}{c}{ Question } & $\begin{array}{c}\text { Agree } \\
(\%)\end{array}$ & $\begin{array}{c}\text { OK } \\
(\%)\end{array}$ & $\begin{array}{c}\text { Disagree } \\
(\%)\end{array}$ \\
\hline $\begin{array}{l}\text { You can easily locate the drug in- } \\
\text { formation you want }\end{array}$ & 78.89 & 18.89 & 2.22 \\
\hline $\begin{array}{l}\text { The computer system is easy to use } \\
82.22\end{array}$ & 16.67 & 1.11 \\
\hline $\begin{array}{l}\text { As a whole, the computer system is } \\
\text { well designed and helpful }\end{array}$ & 84.44 & 14.45 & 1.11 \\
\hline
\end{tabular}

paper prescription with a 2D barcode issued by clinics to any NHI-contracted pharmacy of their choice. The pharmacist scans in the 2D barcode, validates the prescription, and dispenses the drug.

After performing the SDP function, pharmacists in NHI-contracted pharmacies in Taiwan have the right to fill prescriptions from clinics. For the NHI Program, pharmacists must upload refilled prescription data to the NHI. The pharmacists' activities in NHIcontracted pharmacies have increased. Four additional activities are dispensing of prescriptions, checking prescriptions, managing prescriptions and prescription drugs, and prescription filing. Thus more data must be processed than before in NHI-contracted pharmacies, which is a burden on pharmacists. The 2DBPS will assist pharmacists in their additional tasks. This study found that after using the 2DBPS pharmacists almost all agreed that it can efficiently perform the additional activities.

Most patients seek pharmacies with helpful pharmacists, convenient hours, and good information management. ${ }^{11)}$ Pharmacists can reduce their data processing workload with the 2DBPS and spend more time with customers. The Pharmacy Information System (PIS) is a computer software package designed to meet the needs of pharmacy management, excluding additional prescription management, in Taiwan. All community pharmacies have the PIS but only NHI-contracted pharmacies need additional prescription management. Through the use of the PIS, pharmacists can supervise business activities and receive inputs on how and which OTC medications are used in a pharmacy. 2DBPS is a prescription management system, and 2DBPS and PIS are currently independent. If the 2DBPS is linked with the PIS, pharmacies will have greater ability to manage information and

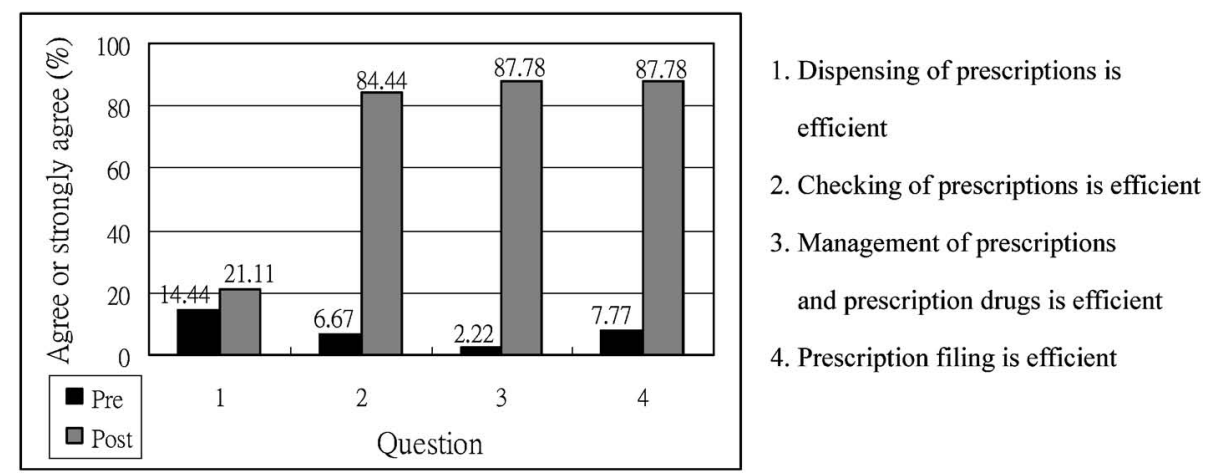

Fig. 1. Comparison of Additional Activities before and after Use of the 2DBPS 
can supervise all business activities of NHI-contracted pharmacies. This will mean more convenience for patients.

Kamei et al. found that insufficient inventories of prescribed drugs influence patient satisfaction, and it will also be important for pharmacies to improve their drug stocks. ${ }^{12)}$ On the 2DBPS, the pharmacist scans in the 2D barcode, validates, and dispenses the drug, and the prescription data are stored in the database for management of prescriptions and prescription drugs. If the 2DBPS is linked with PIS, pharmacies can keep inventory data not only on prescribed drugs but also on OTC preparations so that they can be replenished when inventory is low. Patient satisfaction will improve and pharmacies will be able to lower operating costs.

For optimal healthcare, physicians and pharmacists must team up. Many new high-tech research programs are focusing on how to improve the efficiency and quality of physicians' activities. However, such research is seldom aimed at pharmacists or the management of pharmacies. Our study showed that 2DBPS can improve pharmacists' activities in NHIcontracted pharmacies.

\section{CONCLUSION}

After the NHIP came into effect in March 1995 and SDP in March 1997, pharmacists in NHI-contracted pharmacies have four additional activities. Improving the additional activities is still an issue to be resolved. In this paper, we describe the automatic data collection system 2DBPS for pharmacists. Evaluation of the 2DBPS showed that most pharmacists can use it efficiently to perform their additional activities. Moreover, the easy use of 2DBPS is accepted and appreciated by pharmacists.

As a result of our study, we can conclude that the aim of the 2DBPS is to reduce the workload of pharmacists; on data processing. It will help pharmacists to change their role beyond simple dispensing and data processing. The result is that, pharmacists can shift their role to provide pharmaceutical care services to people.

Acknowledgements We would like to thank the staff of the Information Process Committee of the Taichung City Pharmacist Association for their help in making this study successful. We also thank the reviewers of this journal for their comments in making this paper more readable.

\section{REFERENCES}

1) Chang H. J., Taiwan's National Health Insurance, the 10th Anniversary of Taiwan's National Insurance, 18-19 (2005).

2) The Bureau of National Health Insurance (BNHI), Taiwan: 〈http://www.nhi.gov.tw/.〉 3 September 2007

3) Takagi T., Takayama K., Proceedings of the 124th Annual Meeting of the Pharmaceutical Society of Japan, 29-31 (2004).

4) Smith A. D., Offodile F., Information Management and Computer Security, 10, 109-118 (2002)

5) Marriott M., Sensor Review, 15, 33-35 (1995)

6) Automatic I. D. News Monthly, Cleveland, Ohio, October, 1993.

7) State of Connecticut, USA: 〈http://www.ct. gov $/ \mathrm{dss} / \mathrm{cwp} /$ view.asp? $\mathrm{a}=2349 \& \mathrm{q}=304838$. 1 September 2007

8) SPSS 10.0 User's Guide, SPSS Inc, Chicago, 1999

9) Nunnally J. C., Bernstein I. H., Psychometric Theory, 3rd edition, McGraw Hill, New York, 1994.

10) Jaakko N., Jari F., International Journal of Medical Informatics, 47, 175-182 (1997).

11) Kamei M., Teshima K., Nakamura T., Yakugaku Zasshi, 120, 1185-1191 (2000).

12) Kamei M., Teshima K., Fukushima N., Nakamura T., Yakugaku Zasshi, 121, 215-220 (2001). 